\title{
The Effect of Different Resistance Training Load Schemes on Strength and Body Composition in Trained Men
}

\author{
by \\ Charles Ricardo Lopes ${ }^{1,5}$, Marcelo Saldanha Aoki², Alex Harley Crisp ${ }^{1}$, \\ Renê Scarpari de Mattos², Miguel Alves Lins², Gustavo Ribeiro da Mota³, Brad Jon \\ Schoenfeld ${ }^{4}$, Paulo Henrique Marchetti ${ }^{6}$
}

The purpose of this study was to evaluate the impact of moderate-load (10 RM) and low-load (20 RM) resistance training schemes on maximal strength and body composition. Sixteen resistance-trained men were randomly assigned to 1 of 2 groups: a moderate-load group $(n=8)$ or a low-load group $(n=8)$. The resistance training schemes consisted of 8 exercises performed 4 times per week for 6 weeks. In order to equate the number of repetitions performed by each group, the moderate load group performed 6 sets of 10 RM, while the low load group performed 3 sets of 20 RM. Between-group differences were evaluated using a 2-way ANOVA and independent t-tests. There was no difference in the weekly total load lifted (sets $\times$ reps $\times \mathrm{kg}$ ) between the 2 groups. Both groups equally improved maximal strength and measures of body composition after 6 weeks of resistance training, with no significant between-group differences detected. In conclusion, both moderate-load and low-load resistance training schemes, similar for the total load lifted, induced a similar improvement in maximal strength and body composition in resistance-trained men.

Key words: resistance training, load, volume, body composition, strength.

\section{Introduction}

Resistance training (RT) is a very popular form of physical training for increasing strength and skeletal muscle mass. Adaptive responses to RT include the enhancements of general health, body composition, and athletic performance (Kraemer et al., 2002a). For each purpose, different RT schemes are recommended through manipulation of the acute training variables, such as the load, volume, frequency, rest interval, velocity of execution, range of motion, as well as the choice and order of exercises (American College of Sports Medicine [ACSM], 2009;
Kraemer et al., 2002b). One of these variables, the load, is considered a key variable for the recruitment of motor units (ACSM, 2009; Fry, 2004; Kraemer et al., 2002b).

RT performed with moderate-to-heavy loads is recommended to recruit fast-twitch muscle fibres and to maximize neuromuscular adaptations. In this context, RT with a low (i.e., 3 to 5) number of repetitions maximum (RM) and intermediate (i.e., 9 to 11) RM is considered an appropriate stimulus to increase strength and skeletal muscle mass (Campos et al., 2002).

\footnotetext{
1 - Human Performance Research Group - Methodist University of Piracicaba (UNIMEP) Piracicaba, Sao Paulo, Brazil.

2 - School of Arts, Sciences and Humanities, University of Sao Paulo (USP), Sao Paulo, Brazil.

3 - Department of Sports Sciences - Post-Graduation Program in Physical Education, Federal University of Triangulo Mineiro (UFTM), Uberaba, Minas Gerais, Brazil.

4 - Department of Health Sciences, CUNY Lehman College, Bronx, New York, USA.

5 - Adventist Faculty of Hortolandia (UNASP), Hortolandia, Sao Paulo, Brazil.

6- Department of Kinesiology, California State University, Northridge, CA, USA.
} 
Alternatively, RT performed with a higher number of RM (i.e., 20 to 28 ) is well recognized to increase muscular endurance (Campos et al., 2002).

Recent evidence shows that low load RT performed to volitional muscular failure, defined as the inability to complete a concentric muscle action during exercise, can be as effective as high load RT for inducing skeletal muscle hypertrophy (Mitchell et al., 2012; Ogasawara et al., 2013; Schoenfeld et al., 2015; Gołaś et al., 2016, 2017; Van Roie et al., 2013). It is speculated that a RT scheme performed to volitional muscular failure increases muscle fibre recruitment (Burd et al., 2012) and metabolic stress (Schoenfeld, 2010), thereby promoting favourable muscular adaptations. Thus, lower load RT performed to volitional muscular failure could be an alternative strategy when designing RT programs for skeletal muscle hypertrophy.

Another training variable, the total load lifted (TLL), calculated as the product of the number of sets, repetitions, and the load lifted in each exercise $(\mathrm{kg})$, is also considered to be a key variable of RT programs (ACSM, 2009; Kraemer, 2002b). Despite the relevance of TLL, to date, there is a lack of studies investigating the effect of RT schemes performed with different loading intensities and similar TLL on training-induced adaptations (e.g., strength, body composition, muscle hypertrophy). Additionally, the majority of previous studies had employed untrained subjects, thereby limiting the ability to generalize to those with RT experience. Therefore, the purpose of this study was to evaluate the impact of a moderate-load (ML - $10 \mathrm{RM}$ ) and a low-load (LL - 20 RM) RT scheme on maximal strength and body composition in trained subjects. The initial research hypothesis stated that body composition change and strength gain would be similar comparing the ML and LL schemes performed with similar TLL in trained subjects.

\section{Methods}

\section{Participants}

Sixteen young, recreationally resistancetrained men volunteered to participate in this study. The subjects were pair-matched according to baseline strength and then randomly assigned to 1 of 2 groups ( $n=8$ per group): a group that trained using moderate loads (ML - $10 \mathrm{RM}$ ) (age,
$24.6 \pm 5.8$ years; body height, $175.4 \pm 5.3 \mathrm{~cm}$; body mass: $77.8 \pm 11.72 \mathrm{~kg}$, RT experience, $2.3 \pm 1.4$ years) or a group that trained using light loads (LL - $20 \mathrm{RM}$ ) (age, $28.6 \pm 6.0$ years; body height, $178.3 \pm 7.1 \mathrm{~cm}$; body mass: $85.2 \pm 13.2 \mathrm{~kg}$, RT experience, $3.1 \pm 1.6$ years). There was no significant between-group difference at baseline for age $(p=0.17)$, body mass $(p=0.23)$, height $(p=$ $0.34)$, or training experience $(p=0.29)$. Subjects did not follow a periodized training program and reported their RT programs to be 3 to 5 sets of 8 to 12 repetitions.

All of the participants signed an informed consent document after being informed about the research and experimental protocol. This study was approved by the Research Ethics Committee of the Methodist University of Piracicaba (protocol $n^{-}$19/13). The following inclusion criteria were applied: (a) a minimum of 1 year of continuous experience in RT training at least 3 times a week; (b) no previous injuries that might interfere with the study; (c) competent performance of the bench press and back squat exercises; (d) no use of ergogenic supplements.

\section{Procedures}

A longitudinal, randomized study design was used to assess the effects of 2 different RT schemes on maximal strength and body composition in resistance-trained men. In the week prior to the RT intervention, baseline maximum voluntary muscle strength for each subject was determined across 2 days (i.e., test and re-test, separated by a 24-hour interval) using the 1 repetition maximum (1 RM) test for the bench press and back squat exercises. Additionally, subjects were trained and instructed to record their dietary intake. Body composition assessment was performed 3 days after the final baseline strength test session and, thereafter, subjects began the RT program. The RT program lasted 6 weeks and the TLL was calculated for every exercise session. At the end of the study period, muscle strength tests and measurement of body composition were repeated.

\section{Repetition Maximum Strength Test}

Maximum muscle strength was assessed by the $1 \mathrm{RM}$ test using the bench press and back squat exercises (Charro et al., 2011). Briefly, subjects performed a warm up of 2 sets of 10 repetitions at approximately 40 to $60 \%$ of their estimated $1 \mathrm{RM}$ load before each test protocol. 
After a 5 min rest period, subjects were instructed to perform a single repetition of the test exercise to failure. In subsequent attempts, the load was repeatedly adjusted by 5 to $10 \%$ until the subject was unable to complete the exercise through the full range of motion using only the movement indicated by the exercise itself. For the $1 \mathrm{RM}$ bench press test, subjects were instructed to maintain a 5-point body contact (i.e., head, back and hips were in contact with the bench, and both feet were in contact with the floor) while lifting and lowering the bar to touch the chest. For the 1 RM back squat test, subjects were instructed to perform the movement from standing to $90^{\circ}$ at knee flexion. A researcher controlled the range of motion to validate the movement. Two spotters were present to provide verbal encouragement and to ensure subjects' safety. Each test was performed with a maximum of 5 attempts, with rest intervals of 3 to 5 minutes between each attempt. The $1 \mathrm{RM}$ was considered the highest external load lifted. The $1 \mathrm{RM}$ strength for the bench press and squat exercises showed high testretest reliability (intra-class correlation coefficients $=0.98$ and 0.92 , respectively).

\section{Body Composition}

Body composition, hereby defined as body fat mass and fat-free mass, was estimated by skinfold thickness measurements using a Lange skinfold calliper. The measurements were taken from the subjects' right side at the following 7 sites described by Harrison et al. (1998): subscapular, triceps, pectoral, mid-axillary, suprailiac, abdominal, and anterior mid-thigh. Briefly, subcutaneous fat skinfold was pinched between the thumb/forefinger and pulled away slightly from the underlying muscle before applying the callipers. Each skinfold site was measured 3 times by the same experienced researcher and the mean of these values was used for analysis. Body mass was measured to the nearest $0.1 \mathrm{~kg}$ with a mechanical scale (Welmy). Body density was estimated using the formula proposed by Jackson and Pollock (1978), and body fat was calculated with the equation from Siri (1993).

\section{Estimate of Food Intake}

Dietary nutrient intake was assessed by 24-hour food recalls on 2 non-consecutive weekdays and 1 weekend day. Subjects were instructed to record in detail: time of consumption, types and quantity of food consumed over a $24 \mathrm{~h}$ period. The quantity of food was recorded in cooking units (spoons, cups, and glass) and converted into grams. Energy intake (macronutrients) was estimated using NutWin software (UNIFESP, Sao Paulo, Brazil).

\section{Resistance Training Schemes}

The subjects performed 6 weeks of RT at a frequency of 4 sessions per week (Monday, Tuesday, Thursday, and Friday). Subjects in the ML group performed 6 sets of $10 \mathrm{RM}$ with $1 \mathrm{~min}$ rest intervals between sets and $3 \mathrm{~min}$ rest intervals between exercises. Subjects in the LL group performed 3 sets of $20 \mathrm{RM}$, with 1 min rest intervals between sets and $3 \mathrm{~min}$ rest intervals between exercises. The loads for $10 \mathrm{RM}$ and 20 RM were determined and adjusted during each exercise and each set. Subjects were verbally encouraged and motivated to perform each set until volitional fatigue. All RT sessions were supervised by personal trainers to ensure that the target RM was achieved. If assistance was provided to subjects during the exercise to complete the proposed RM, the external load was adjusted for the subsequent set to maintain the $\mathrm{RM}$ zone (10 RM or $20 \mathrm{RM}$ ) and total number of repetitions per exercise (60 repetitions). Sessions were rotated between lower and upper body exercise selection, as shown in Table 1, to promote adequate recovery of major muscle groups between sessions. No injuries were reported and adherence to the program was $100 \%$ for both groups.

\section{Statistical Analysis}

Normality and homogeneity of variance of the data were confirmed by the Shapiro-Wilk and Levene's tests, respectively. A 2-way repeated measures analysis of variance (ANOVA) was performed to compare the change in outcome measures over time between the groups. For significant interaction effects, a Bonferroni posthoc test was performed. Independent $t$-tests were used to compare the baseline and percentage change (\% change) pre to post-training. Test-retest reliability (ICC) was calculated for 1 RM tests. A Cohen's formula (Mean group 1-Mean group 2/pooled standard deviation) was used to calculate effect size (ES). According to Rhea (2004), ES for recreational RT is considered to be trivial for ES values $<0.35$, small for ES values between 0.35 and 0.80 , moderate for ES values between 0.80 and 1.50 , and large for ES values $>1.5$. The level of 
significance was set at $5 \%$ for all analyses. All data are presented as means \pm standard deviation.

\section{Results}

Energy and macronutrient intake did not differ pre and post-study among subjects in the ML and LL groups ( $p>0.05$; Table 2).

There was a significant effect of time $(\mathrm{F}=$ 114.90; $p<0.001$ ) for the TLL; however, there was no group interaction effect $(\mathrm{F}=2.14 ; p=0.16$; Table 3). Body composition variables and $1 \mathrm{RM}$ strength results are reported in Table 4 . There was a significant group by time interaction for body mass $(\mathrm{F}=5.25 ; p=0.03)$, body fat percentage $(\mathrm{F}=$ $51.58 ; p<0.001)$, fat-free mass ( $\mathrm{F}=176.5 ; p<0.001)$, fat mass $(\mathrm{F}=26.48 ; p<0.001), 1 \mathrm{RM}$ bench press $(\mathrm{F}=352.2 ; p<0.001)$, and $1 \mathrm{RM}$ squat $(\mathrm{F}=285.0 ; p<$ 0.001). However, there was no significant interaction effect $(p>0.05)$ between ML and LL groups for all dependent variables. A moderate ES of the training group was calculated for body mass and the 1 RM bench press.

\section{Table 1}

The choice and order of exercises for $A$ and $B$ sessions

\begin{tabular}{ll}
\hline \multicolumn{1}{c}{ Exercise Session A } & \multicolumn{1}{c}{ Exercise Session B } \\
\hline 1 - Bench Press & 1- Back lat pull-down \\
2 - Inclined Bench Press & 2- One-arm dumbbell row \\
3 - Fly & 3- Row with open grip \\
4 - Standing biceps curls & 4- Triceps overhead extension \\
5 - Concentrated biceps curls & 5- Triceps pushdown \\
6- Back Squat & 6- Leg curl \\
7 - Leg Press $45^{\circ}$ & 7- Calf raise \\
8- Abdominal crunch & 8- Abdominal crunch \\
\hline
\end{tabular}

Table 2

Estimated energy and macronutrient intake in the pre-and post-training period for the $M L$ and $L L$ groups.

\begin{tabular}{cccc}
\hline Variables & & ML & LL \\
\hline Energy Intake (kcal) & Pre & $2938.1 \pm 350.5$ & $3029.26 \pm 360.4$ \\
& Post & $3051.9 \pm 322.3$ & $3199.51 \pm 420.7$ \\
Carbohydrate (\%) & Pre & $47.0 \pm 1.3$ & $55.65 \pm 0.4$ \\
& Post & $49.4 \pm 0.5$ & $53.83 \pm 3.9$ \\
Fat (\%) & Pre & $24.0 \pm 1.2$ & $25.92 \pm 1.3$ \\
& Post & $25.6 \pm 1.2$ & $26.19 \pm 4.4$ \\
Protein $(\%)$ & Pre & $25.6 \pm 2.6$ & $19.66 \pm 1.9$ \\
& Post & $24.8 \pm 1.0$ & $22.33 \pm 2.2$ \\
\hline
\end{tabular}






Table 4

Body composition and 1 repetition maximum (1 RM) test for the ML and LL groups.

\begin{tabular}{|c|c|c|c|c|c|}
\hline Variables & & ML & LL & $\begin{array}{l}\text { Effect } \\
\text { Size }\end{array}$ & \\
\hline \multirow[t]{3}{*}{ Body Mass (kg) } & Pre & $77.9 \pm 11.7$ & $85.2 \pm 13.2$ & 0.58 & Small \\
\hline & Post & $79.9 \pm 11.7^{*}$ & $86.5 \pm 12.6^{*}$ & 0.86 & Moderate \\
\hline & $\% \Delta$ & $2.7 \pm 1.3$ & $1.7 \pm 1.4$ & 0.78 & Small \\
\hline \multirow[t]{4}{*}{ Body Fat (\%) } & Pre & $15.8 \pm 6.4$ & $18.6 \pm 5.0$ & 0.49 & Small \\
\hline & Post & $14.5 \pm 5.7^{*}$ & $16.9 \pm 4.5^{*}$ & 0.46 & Small \\
\hline & $\% \Delta$ & $-7.3 \pm 5.1$ & $-9.2 \pm 3.1$ & 0.43 & Small \\
\hline & Pre & $65.0 \pm 7.8$ & $69.1 \pm 9.9$ & 0.46 & Small \\
\hline \multirow[t]{2}{*}{ Fat Free Mass (kg) } & Post & $68.1 \pm 8.1^{*}$ & $71.6 \pm 9.6^{*}$ & 0.40 & Small \\
\hline & $\% \Delta$ & $4.7 \pm 1.0$ & $3.71 \pm 1.7$ & 0.74 & Small \\
\hline \multirow[t]{3}{*}{ Fat Mass (kg) } & Pre & $12.6 \pm 6.2$ & $16.1 \pm 6.0$ & 0.56 & Small \\
\hline & Post & $11.9 \pm 5.8^{*}$ & $14.8 \pm 5.4^{*}$ & 0.51 & Small \\
\hline & $\% \Delta$ & $-4.8 \pm 6.3$ & $-7.7 \pm 2.8$ & 0.60 & Small \\
\hline \multirow[t]{3}{*}{$\begin{array}{l}1 \text { RM Bench Press } \\
(\mathrm{kg})\end{array}$} & Pre & $90.4 \pm 19.1$ & $94.4 \pm 22.5$ & 0.19 & Trivial \\
\hline & Post & $96.0 \pm 19.1^{*}$ & $98.9 \pm 22.5^{*}$ & 0.14 & Trivial \\
\hline & $\% \Delta$ & $6.4 \pm 2.5$ & $4.9 \pm 1.2$ & 0.81 & Moderate \\
\hline \multirow[t]{3}{*}{1 RM Squat (kg) } & Pre & $126.6 \pm 39.0$ & $121.5 \pm 28.9$ & 0.15 & Trivial \\
\hline & Post & $138.2 \pm 37.9^{*}$ & $130.9 \pm 29.1^{*}$ & 0.22 & Trivial \\
\hline & $\% \Delta$ & $10.5 \pm 7.0$ & $8.0 \pm 1.9$ & 0.49 & Small \\
\hline
\end{tabular}

${ }^{*}$ Significant difference $(p<0.05)$ compared to pre-training values. 


\section{Discussion}

The main finding of the present study was the absence of a significant difference between the ML and LL training groups on measures of body composition and maximal strength following 2 different 6-week RT programs similar for the TLL. These results support the initial study hypothesis that different training schemes, with similar TLL, would evoke similar outcomes in body composition and muscle strength.

Equating RT volume provides the best model to compare different training schemes. In the current study, the ML and LL schemes were similar for the TLL by having subjects in each group completing the same number of RM per exercise (i.e., $60 \mathrm{RM}$ ) but with different loading zones: ML, $6 \times 10 \mathrm{RM}$ and LL, $3 \times 20 \mathrm{RM}$.

Previous studies have shown the magnitude of muscle damage (Barroso et al., 2010; Charro et al., 2011; Paschalis et al., 2005; Uchida et al., 2009) and hormonal response to RT (Crewther et al., 2008; Uchida et al., 2009) to be similar for different schemes when the TLL was comparable between programs. Therefore, acute mechanical and hormonal responses to resistance exercise are dependent on the total external work performed in the exercise session, regardless of the training intensity. The TLL is a key variable of resistance exercise prescription that should be monitored and progressively adjusted during the training program to maximize neuromuscular adaptations.

In the current study, the TLL was significantly increased for both the ML and LL groups (Table 3), despite training in different loading zones (10 RM vs. $20 \mathrm{RM})$, with no significant between-group differences. ML and LL groups increased their TLL by $19.9 \%$ and $20.7 \%$, respectively, from week 1 to 6 of the RT period. It should be noted that all RT sessions were supervised by personal trainers, who instructed and motivated each subject to gradually increase the exercise load during the 6-week training period. Therefore, both RT schemes produced a progressive increase in the total amount of weight lifted and were effective in increasing strength.

With respect to maximal strength, both groups increased 1 RM strength on both the bench press and back squat exercises, with no betweengroup differences. This result is contrary to previous studies that have reported a higher strength gain with high- versus low-load training in untrained subjects (Campos et al., 2002; Mitchell et al., 2012; Ogasawara et al., 2013; Van Roie et al., 2013). The discrepancy between these studies and current results could be explained by (a) participants' training status (i.e., untrained vs. resistance-trained men); (b) the possible need for a higher external training load to increase the magnitude of neuromuscular adaptations; and/or (c) the short duration of the RT period used in this study (i.e., 6 weeks).

The early phase of strength adaptation in untrained individuals is largely attributed to neural mechanisms, while the increase in skeletal muscle hypertrophy occurs over a longer period of time (Moritani and deVries, 1979). Accordingly, previous studies have shown training at moderate-to-high loads to be superior to increase strength in untrained individuals (Campos et al., 2002; Mitchell et al., 2012; Ogasawara et al., 2013; Van Roie et al., 2013). In this study, all subjects were experienced with RT, performing 8 to 12 repetitions on a regular training basis. Therefore, it is possible that neural adaptations in trained men would not be as evident as in untrained peers. Alternatively, the novelty of the light-load stimulus might have elicited a training effect in the LL group that enhanced strength. Recently, Schoenfeld et al. (2015) found that training at 10 $\mathrm{RM}$ produced a significantly greater increase in maximal strength compared to an $\sim 30$ RM protocol in well-trained young men, even though the training volume was markedly higher in the light-load condition. A potential reason for the discrepancy between findings is that the study by Schoenfeld et al. (2015) lasted 8 weeks compared to only 6 weeks in the present study. Indeed, the current results did show a relative, albeit nonsignificant, superiority for both the bench press (6.4\% versus $4.9 \%$ ) and squat $(9.1 \%$ versus $7.6 \%)$ in the ML compared to the LL group, respectively, with a calculated ES of 0.81 . It is possible that the changes could have become significant had the protocol been continued for a longer period of time. It also should be noted that the light-load condition in the Schoenfeld et al.'s study (2015) was carried out at a substantially lower intensity of the load compared to that in the present study (30 RM versus $20 \mathrm{RM}$, respectively), which likely influenced the magnitude of strength gains in the respective studies.

There is consensus that high-intensity and 
low-volume RT schemes stimulate high threshold motor units thus enhancing maximal strength development (Cormie et al., 2011). In support of this assumption, Schoenfeld et al. (2014) showed that a higher load powerlifting-type training scheme (i.e., $7 \times 3 \mathrm{RM}$ ) produced a superior increase in $1 \mathrm{RM}$ bench press and back squat performance compared to a volume-equated to moderate load (i.e., $3 \times 10 \mathrm{RM}$ ) bodybuilding training scheme in trained subjects, over the 8week training period. These data suggest that a higher load RT program (i.e., 3 RM) is likely to be necessary to maximize $1 \mathrm{RM}$ strength in welltrained subjects.

It is well established that RT programs can induce a significant improvement in body composition variables (Crewther et al., 2013; Kraemer et al., 2000; Schmitz et al., 2003). With respect to body composition analysis in this study, both groups (ML and LL) increased body mass and fat-free mass and decreased percent body fat and fat mass, with no between-group differences noted (Table 4). The current results are in contrast to those of Holm et al. (2008) who reported a significantly greater increase in muscle cross-sectional area when training at $70 \%$ of $1 \mathrm{RM}$ versus $15 \%$ of $1 \mathrm{RM}$ for 12 weeks on a workmatched basis. In the Holm et al.'s (2008) study, participants did not train to muscular failure, an important difference from the RT schemes used in this study, in which participants performed repetitions to volitional fatigue. Given that fatiguing contractions are deemed essential to elicit activation of high-threshold motor units during submaximal resistance exercise (Adam and De Luca, 2003; Houtman et al., 2003), this provides a logical explanation for differences in the outcome between the 2 studies, and reinforces the importance of high intensities of effort when training with low-loads.

In agreement with the current results, a previous study using different volume-equated RT loading schemes (load ranging - 4 RM, 6 RM and $8 \mathrm{RM}$ ) reported no significant difference in body composition (Buford et al., 2007). Therefore, equating the TLL is likely to be a major factor in determining effects of RT on body composition of trained individuals. The current results also are consistent with those of Schoenfeld et al. (2015), who found a similar increase in thickness of the elbow flexors, elbow extensors, and quadriceps femoris for well-trained individuals when training at $10 \mathrm{RM}$ and $30 \mathrm{RM}$. The present study expands on these findings to show that resistancetrained men experience a similar increase in whole body lean mass, irrespective of the loading zone employed, when resistance training schemes are carried out to muscular failure.

A number of studies failed to observe a significant effect of short-term RT programs (i.e., $<12$ weeks) on body composition (Apel et al., 2011; Monteiro et al., 2009; Prestes et al., 2009; Rhea et al., 2002). The improvement in body composition produced by the 6-week RT program in this study might be due to the maintenance of energy intake and high protein consumption throughout the study. All participants received nutritional counselling, and estimation of energy and macronutrient intake confirmed that both groups did not change their intake and that there was no influence of the different RT schemes (Table 2). Thus, it is suggested that, to effectively use shortterm RT programs to improve body composition, a dietary control should be included as a component of the program.

A limitation of the current study was that while the skinfold technique is a valid method for assessing global changes in fat mass and fat-free mass (Bentzur et al., 2008), it does not provide a direct measurement of muscle mass. Modalities such as magnetic resonance imaging, computerized tomography, and ultrasound would therefore provide better insight into specific hypertrophic development in the trained muscles.

In conclusion, ML (10 RM) and LL (20 RM) volume-equated RT schemes promoted a similar change in body composition and strength in resistance-trained men. These results suggest that performing repetitions to volitional muscular failure is the primary factor in promoting muscular adaptations and the variation of loading schemes has a secondary role in this regard in resistance-trained men. Further studies are necessary to investigate the effects of longer periods of RT (i.e., $>6$ weeks) using direct methods of assessing muscle hypertrophy. 


\section{Acknowledgements}

We gratefully acknowledge the subjects who dedicated a great deal of time to participate in this study.

\section{References}

Adam A, De Luca CJ. Recruitment order of motor units in human vastus lateralis muscle is maintained during fatiguing contractions. J Neurophysiol, 2003; 90: 2919-2927

American College of Sports Medicine. American College of Sports Medicine position stand. Progression models in resistance training for healthy adults. Med Sci Sports Exerc, 2009; 41: 687-708

Apel JM, Lacey RM, Kell RT. A comparison of traditional and weekly undulating periodized strength training programs with total volume and intensity equated. J Strength Cond Res, 2011; 25: 694-703

Barroso R, Rochel H, Ugrinowitsch C, Araújo R, Nosaka K, Tricoli V. Effect of eccentric contraction velocity on muscle damage in repeated bouts of elbow flexor exercise. Appl Physiol Nutr Metab, 2010; 35: 534540

Bentzur KM, Kravitz L, Lockner DW. Evaluation of BOD POD for estimating percent body fat in collegiate track and field female athletes: a comparison of four methods. J Strength Cond Res, 2008; 22: 1985-1991

Buford TW, Rossi SJ, Smith DB, Warren AJ. A comparison of periodization during nine weeks with equated volume and intensity for strength. J Strength Cond Res, 2007; 21: 1245-1250

Burd NA, Mitchell CJ, Churchward-Venne TA, Phillips SM. Bigger weights may not beget bigger muscles: evidence from acute muscle protein synthetic responses after resistance exercise. Appl Physiol Nutr Metab, 2012; 37: 551-554

Campos GE, Luecke TJ, Wendeln HK, Toma K, Hagerman FC, Murray TF, Ragg KE, Ratamess NA, Kraemer WJ, Staron RS. Muscular adaptations in response to three different resistance-training regimens: specificity of repetition maximum training zones. Eur J Appl Physiol, 2002; 88: 50-60

Charro MA, Aoki MS, Nosaka K, Foschini D, Jr Figueira A, Bacurau RF. Comparison between multiple sets and half-pyramid resistance exercise bout for the muscle damage profile. Eur J Sports Sci, 2011; 12: $249-254$

Cormie P, McGuigan MR, Newton RU. Developing maximal neuromuscular power: part 2 - training considerations for improving maximal power production. Sports Med, 2011; 41: 125-146

Crewther B, Cronin J, Keogh J, Cook C. The salivary testosterone and cortisol response to three loading schemes. J Strength Cond Res, 2008; 22: 250-255

Crewther BT, Heke TL, Keogh JW. The effects of a resistance-training program on strength, body composition and baseline hormones in male athletes training concurrently for rugby union 7's. J Sports Med Phys Fitness, 2013; 53: 34-41

Drinkwater EJ, Lawton TW, Lindsell RP, Pyne DB, Hunt PH, Mckenna MJ. Training leading to repetition failure enhances bench press gains in elite junior athletes. J Strength Cond Res, 2005: 19: 382-388

Fry AC. The role of resistance exercise intensity on muscle fibre adaptations. Sports Med, 2004; 34: 663-679

Gołaś A, Maszczyk A, Zajac A, Mikołajec K, Stastny P. Optimizing post activation potentiation for explosive activities in competitive sports. J Hum Kinet. 2016; 52: 95-106

Gołaś A, Maszczyk A, Pietraszewski P, Stastny P, Tufano JJ, Zając A. Effects of Pre-exhaustion on the Patterns of Muscular Activity in the Flat Bench Press. J Strength Cond Res. 2017; 31(7): 1919-1924

Harrinson GG, Buskirk ER, Lindsay Cater JE, Johnston FE, Lohman TG, Pollock ML, Roche AF, Wilmore J. Skinfold thickness and measurement technique. In: Lohman TG, Roche AF, Martorell R eds. Champaign, 
IL: Human Kinetics, 5-70; 1988

Holm L, Reitelseder S, Pedersen TG, Doessing S, Petersen SG, Flyvbjerg A, Andersen JL, Aagaard P, Kjaer M. Changes in muscle size and $\mathrm{MHC}$ composition in response to resistance exercise with heavy and light loading intensity. J Appl Physiol (1985), 2008; 105: 1451-1461

Houtman CJ, Stegeman DF, Van Dijk JP, Zwarts MJ. Changes in muscle fibre conduction velocity indicate recruitment of distinct motor unit populations. J Appl Physiol (1985), 2003; 95: 1045-1054

Jackson AS, Pollock ML. Generalized equations for predicting body density of men. 1978. Br J Nutr, 2004; 40: 497-504

Kraemer WJ, Ratamess N, Fry AC, Triplett-McBride T, Koziris LP, Lynch JM, Fleck SJ. Influence of resistance training volume and periodization on physiological and performance adaptations in collegiate women tennis players. Am J Sports Med, 2000; 28: 626-633

Kraemer WJ, Ratamess NA, French DN. Resistance training for health and performance. Curr Sports Med Rep, 2002a; 1: 165-171

Kraemer WJ, Adams K, Cafarelli E, Dudley GA, Dooly C, Feigenbaum MS, Fleck AC, Hoffman JR, Newton RU, Potteiger J, Stone MH, Ratamess NA, Triplett-McBride T, American College of Sports Medicine. American College of Sports Medicine position stand. Progression models in resistance training for healthy adults. Med Sci Sports Exerc, 2002b; 34: 364-380

Loenneke JP, Wray ME, Wilson JM, Barnes JT, Kearneym ML, Pujol TJ. Accuracy of field methods in assessing body fat in collegiate baseball players. Res Sports Med, 2013; 21: 286-291

Mitchell CJ, Churchward-Venne TA, West DW, Burd NA, Breen L, Baker SK, Phillips SM. Resistance exercise load does not determined training-mediated hypertrophic gains in young men. J Appl Physiol (1985), 2012; 113: 71-77

Monteiro AG, Aoki MS, Evangelista AL, Alveno DA, Monteiro GA, Piçarro Ida C, Ugrinowitsch C. Non linear periodization maximizes strength gains in split resistance routines. J Strength Cond Res, 2009; 23: 1321-1326

Moritani T, deVries HA. Neural factors versus hypertrophy in the time course of muscle strength gain. Am J Phys Med, 1979; 58: 115-130

Ogasawara R, Loenneke JP, Thiebaud RS, Abe T. Low-load bench press training to fatigue results in muscle hypertrophy similar to high-load bench press training. Int J Clinical Med, 2013; 4: 114-121

Paschalis V, Koutedakis Y, Jamurtas AZ, Mougios V, Baltzopoulos V. Equal volumes of high and low intensity of eccentric exercise in relation to muscle damage and performance. J Strength Cond Res, 2005; 19: $184-188$

Prestes J, Frollini AB, de Lima C, Donatto FF, Foschini D, de Cássia Marqueti R, Figueira A Jr, Fleck SJ. Comparison between linear and daily undulating periodized resistance training to increase strength. $J$ Strength Cond Res, 2009; 23: 2437-2442

Rhea MR, Ball SD, Phillips WT, Burkett LN. A comparison of linear and daily undulating periodized programs with equated volume and intensity for strength. J Strength Cond Res, 2002; 16: 250-255

Rhea MR. Determining the magnitude of treatment effects in strength training research through the use of the effect size. J Strength Cond Res, 2004; 18: 918-920

Schmitz KH, Jensen MD, Kugler KC, Jeffery RW, Leon AS. Strength training for obesity prevention in midlife women. Int J Obes Relat Metab Disord, 2003; 27: 326-333

Schoenfeld BJ. The mechanism of muscle hypertrophy and their application to resistance training. J Strength Cond Res, 2010; 24: 2857-2872 
Schoenfeld BJ, Ratamess NA, Peterson MD, Contreras B, Sonmez GT, Alvar BA. Effects of different volume-equated resistance training loading strategies on muscular adaptations in well-trained men. $J$ Strength Cond Res, 2014; 28: 2909-2918

Schoenfeld BJ, Peterson MD, Ogborn D, Contreras B, Sonmez GT. Effects of Low- Versus High-Load Resistance Training on Muscle Strength and Hypertrophy in Well-Trained Men.J Strength Cond Res, 2015; 29: 2954-2963.

Siri WE. Body composition from fluid space and density: analysis of methods. 1961. Nutrition, 1993; 9: 480491

Uchida MC, Nosaka K, Ugrinowitsch C, Yamashita A, Martins E Jr, Moriscot AS, Aoki MS. Effect of bench press exercise intensity on muscle soreness and inflammatory mediators. J Sports Sci, 2009; 27: 499-507

Uchida MC, Crewther BT, Ugrinowitsch C, Bacurau RF, Mariscot AS, Aoki MS. Hormonal responses to different resistance exercise schemes of similar total volume. J Strength Cond Res, 2009; 23: 2003-2008

Van Roie E, Delecluse C, Coudyzer, W, Boonen S, Bautmans I. Strength training at high versus low external resistance in older adults: effects on muscle volume, muscle strength, and force-velocity characteristics. Experimental Gerontology, 2013; 48: 1351-1361

\section{Corresponding author:}

\section{Charles R. Lopes}

Methodist University of Piracicaba (UNIMEP) - Campus Taquaral

Rodovia do Açucar, km 156, s/n. Pitacicaba-SP, Brazil

Phone: (19) 3124-1558

Email: chrlopes@uimep.br 